\title{
An Energy Efficient Optimal Model Using Social Spider Optimization Algorithm
}

\author{
Pavithra G S ${ }^{1, *}$, Babu N V ${ }^{2}$, Puttamadappa C. ${ }^{3}$ \\ ${ }^{1}$ Department of Computer Science \& Engineering, SJB Institute of Technology, \\ Bengaluru, India \\ ${ }^{2}$ Department of Electrical \& Electronics Engineering, SJB Institute of Technology, \\ Bengaluru, India \\ ${ }^{3}$ Dayanandasagar University, Bangalore, India \\ pavi.pgs@gmail.com,dr.babunv@gmail.com,puttamadappa-ece@dsu.edu.in
}

\begin{abstract}
Wireless Sensor Networks (WSNs) constitute Sensor Nodes (SNs) that collect and process the information present in the physical environment through the network area to the receiver. Each sensor node is powered with respect to non-replicable batteries, which conserve the energy sources from the sensor nodes. The challenge in designing WSNs for routing an efficient model is to preserve the power using the nodes. To solve such an issue, a clustering approach for energy preservation in WSN. Initially, the SNs are deployed randomly over a network where the SNs' locations are given as input to the clustering algorithm. The Social Spider Optimization Algorithm (SSOA) based Clustering is used to Cluster Head Selection between the nodes which reduces the energy consumption and traffic during the transmission of data from source to destination. The location of the selected Cluster Heads (CHs) is given as input to the SSOA. Additionally, the number of packets from the BS is received which increases by considering the energy in the fitness function. The proposed SSOA showed improvement in energy consumption of $20 \%$ when compared to the existing GAPSO-H, MW-LEACH optimization models.
\end{abstract}

Keywords: Cluster head selection, clustering, energy consumption, routing, social spider optimization algorithm, wireless sensor networks. 


\section{Introduction}

Recently, the WSNs operates a wide number of tiny and low-powered SNs that are present in an unreliable environment to utilize network components for communication (Pitchaimanickam and Murugaboopathi, 2020). The wide variety range of applications employed using WSNs includes national defense, military, traffic management, monitoring the environment, manufacturing, health care, and disaster prevention applications (Zhang et al., 2020). However, monitoring the WSNs requires deployment for a huge sensor node which limits in terms of communication capacities, computational, reduce costs, and storage (Mehta and Saxena, 2020). This limitation is due to the randomly deployed nodes that are not monitored and are operated into the structure of the network such as AdHoc. SNs that are utilized in the networks are often disposable and are expected till the energy drains. Thus, the energy source needs to be wisely managed as long as the network lifetime is sustained (Preethiya et al., 2020; Singh, 2020). The transmission power is independently reduced on every node where the proper allocation of resources for each of them should be provided to improve the overall performance of the network (Han, 2020). In few cases like conventional routing approaches, dynamic sensor nodes' nature is restricted as the sensor-based networks which are demanding to design the protocol for environment distribution (Boveiri, 2020; Rao at al., 2017). Therefore clustering approaches were developed to deal the problems such as network scalability and energy efficiency was attained among researchers that are categorized as centralized and distributed methods. The scalability of the networks is evaluated in terms of time scheduling, switching between the rest and active states within a periodic interval (Han et al, 2017) to improve the performance of a network. In the present research work, the main aim is to introduce WSNs as one of the important concerns for improving energy efficiency and saves unnecessary consumption of power among the nodes (Jha and Eyong, 2018). The group of nodes forms clusters where the process of clustering will help to preserve the power of nodes to find an optimal solution to select each node. Each of the clusters will be having Cluster Head $(\mathrm{CH})$ and each cluster has its properties that will be functioned in the network area. The energy provided by both the sensor nodes is saved in such a manner that are aggregated by data from the $\mathrm{CH}$ are utilized to energy saving of the nodes.

Various existing methodologies were developed like Particle Swarm Optimization (PSO), Genetic Algorithm (GA), Cuckoo Search Algorithm (CSA), Artificial Bee Colony (ABC), and Social Network Optimization (SNO). The particles are considered to find the best position to prevent the premature convergence which identified the sub-optimal solutions for balancing the clusters. Based on these characteristics the SSOA algorithm has been utilized to solve a wide range of optimization problems where the clustering algorithms are compared to state-of-art techniques. The existing models failed to optimize the end-to-end route 
discovery results with higher energy consumption with the communication cost in a network field. The data dissemination in the WSN will face challenges as the sensor nodes are a part of the constrained resources. The present research carries out the process for $\mathrm{CH}$ selection for optimizing the data during transmission between the SNs even for the process of node masking.

The structure of the research paper is presented as follows: Section 2 describes the various clustering and optimization algorithms with their variants for energy consumption minimization. Section 3 discussed the optimization problem that occurred in the existing approaches and Section 4 discusses the preliminaries for the proposed work and section 5 describes the proposed SSOA algorithm and its role in reducing the energy consumption during transmissions. Section 6 describes the results and discussion for the research work that includes quantitative and comparative analysis. Section 7 presents the conclusion and future work of this research work.

\section{Related Works}

The Energy efficiency for WSNs is important which defines the lifetime for the network. The efficiency of each energy node is provided for certain nodes but in few scenarios, the power was limited in the batteries. The nodes utilize the power effectively in an intelligent manner makes longer live. Numerous researches effectively utilized the power of intelligence to make them long-lasting. There are various research investigations observed in the literature that is related to energyeffective routing techniques. The energy efficiency obtained from each node is provided and processed which shows the limited capabilities through processing the functions using the batteries. The usage of available power intelligently is important to make use of energy effective for routing the models. An effective routing mechanism is utilized for energy preservation and related works have been proposed discussed in this section. The existing methods explained in the next section are based on clustering and routing for energy management that worked on the improvement of energy efficiency for WSNs are reviewed as follows:

\subsection{Cluster Head Selection based Approaches}

Haseeb et al. (2017) developed an Adaptive Energy-Aware Cluster (AECR) based routing protocol to improve the performance of a network. The existing models used energy-aware routing protocols showed the unbalance during energy consumption that was inefficient to load the balance to maintain the network lifetime. The major aim of the research was developed to improve the data delivery rate and energy conservation in terms of performance. The developed model used appropriate clusters that are created over the network where the weighted score defines the selection of $\mathrm{CH}$ and $\mathrm{CHs}$ role was shifted frequently to improve the network connectivity was minimized based on the optimal $\mathrm{CH}$ selection. In the 
AECR protocol, the formation of clusters was mainly considering only at the energy node level. Thus, the AECR protocol failed to consider the heterogeneous data generation traffic. Additionally, the multimedia content related to the energy nodes showed a critical challenge when the nodes were provided with limited power and lowered the network efficiency. Therefore, Ezhilarasi and Krishnaveni (2019), developed an Evolutionary Multipath Energy-Efficient Routing (EMEER) protocol that includes CSA which enhanced the network efficiency. As the developed model is based on clustering, an evolutionary algorithm operates in a multi-path which improves the energy efficiency in the network.. The developed CSA creates a cluster in such a way that considered the energy level features and similarity based upon the distance from the node to sink the energy. However, the energy consumption of the network was less than the standard protocols that failed to analyze the throughput of the network. Similarly, Wang and Wang (2019) presented the PEC-Ad hoc On-Demand Distance Vector (AODV) routing protocol to balance the node's energy consumption in the WSN. This message transmitted the information about residual energy to accomplish the network node to obtain the partial energy level. The link information and node's energy consumption were provided to the adjacent nodes by broadcasting the message and the message was used to observe the link information of the node. The energy consumption of the WSN was not analyzed while transmitting the data packets because the PEC-AODV energy-efficient routing protocols failed to assign a proper threshold setting.

Similarly, the selection of cluster heads is also important as the wireless links determined the transmission powers of the sensor nodes. The longer distance for transmissions of data by SNs is not much efficient and thus energy loss occurred. The cluster-based energy-efficient router placement is important for WSNs where the k-means algorithm was utilized to select the cluster heads by Jemal et al. (2018). In the cluster-based energy-efficient router placement scheme where the Kmeans algorithm was used to select the initial $\mathrm{CHs}$ from the network. The calculation of Euclidean distance was used to place the computer-generated nodes (CGNs) in the centroid for the $\mathrm{CH}$ placement. The candidate node was chosen as $\mathrm{CH}$ when the candidate $\mathrm{CH}$ was near the $\mathrm{CGN}$. If there was $\mathrm{CH}$ failure in the WSN, the cluster member with higher energy was considered as $\mathrm{CH}$ which doesn't require any extra communication to accomplish the previous $\mathrm{CH}$ process. However, the direct data transmission between the $\mathrm{CH}$ to a destination was consumed huge energy during data transmission. Similarly, Ya-qiong and Yun-Rui (2016), developed the K-means clustering and Dijkstra routing algorithm (KDUCR) to avoid the communication over a long distance which balance the energy consumption. The K-means clustering has divided the network into $k$ clusters and the $\mathrm{CH}$ was selected from each cluster. Subsequently, the Dijkstra algorithm was used by the sink to identify the shortest path from the $\mathrm{CH}$ to the sink node. The $\mathrm{CH}$ separated the time into different slots based on cluster members where the different 
slots namely TDMA was used to allocate the transmission time slots to each cluster member. The distance to the BS was not considered during the clustering process using the K-means algorithm however the developed KDUCR was not appropriate in a WSN environment because it penalizes an energy node without considering the battery lifetime.

\subsection{Optimization-based Approaches}

The nodes of WSN are battery powered that would create a loss of energy after a certain period, therefore the present research work considered an energy constraint that minimized the consumption of overall energy to maximized the network's lifetime. Maheshwari et al. (2016) developed an efficient energy-based cluster routing approach using Ant Colony Optimization (ACO) and Butterfly Optimization Algorithm (BOA) in WSNs. The developed model selected an optimal route that obtains a residual amount of energy based on the node energy, distance. The residual energy showed better values of performance for the BOA. The BOA showed better performance to test the functions using meta-heuristic optimization approaches which show the challenges when complex optimization problems have occurred. Because of the wireless SNs existence, the batteries extended the survival time as the batteries were not placed in an unattended area. Therefore, Sahoo et al. (2021) developed Genetic Algorithm-Particle Swarm Optimization Hybrid (GAPSO-H) for cluster optimization based on the routing approaches. The developed model performed $\mathrm{CH}$ selection based on the sink mobility for data transmission approach which utilized hybrid approaches for optimizing the nodes that considered GA-PSO for performing the task. The node constraint considered GA-PSO to perform the task whereas the node constraint has no such limitation. The data transmission is performed to a certain round of data for $\mathrm{CH}$ nodes exhausted thus restriction on the energy sources sink with other nodes.

El Khediri et al. (2020) developed a Multiple Weight Low Energy Adaptive Clustering Hierarchy (MW-LEACH) protocol for $\mathrm{CHs}$ selection based on the $\mathrm{CHs}$ distances, residual energy, and optimal member of nodes. In the initial stage, the nodes were selected based on the residual energy which is closer to the density from the cluster center and thus sets up the set of $\mathrm{CH}$ candidates in an initial set. However, the developed model was not suitable to implement an initial set of $\mathrm{CH}$ candidates and was not applied for real testbed sensors for various application domains. Similarly, Prithi and Sumathi (2021) developed an Automata Hybrid Particle Swarm Optimization-Grey Wolf Optimizer (PSO-GWO) for providing secured energy efficiency in WSNs to obtain an Optimal Routing solution. The developed hybrid PSO-GWO efficiently utilized the energy to secure the data transmission based on the augmented path. The developed automated dynamic deterministic Finite Automated (LD2FA) model initiated the learning phase which innovated the dynamic role in an environment. The LD2FA provided the features for learning that accepts a string for hybrid PSO-GWO routed for optimization. However, the 
developed model was dispersed and showed balance when it was subsequently terminated formed clusters further.

\section{Problem Statement}

The WSN model extends the lifetime of WSN nodes and battery nodes are not replaced or recharged again once the nodes are exhausted. The node deployments in WSN are not efficient due to the power demands of nodes creates complexity and power demands during network characterization. An optimal $\mathrm{CH}$ selection is important with respect to multiple criteria scenarios to decide on the challenging task.

Solution: Therefore, the selection of $\mathrm{CH}$ is performed using the proposed SSOA optimization approach that considers the constraints and leftover total energy after each repetitive iteration of the cycle. The minimum energy is considered to select the $\mathrm{CH}$ with high priority. The SSOA algorithm considers the CNs that consist of the highest number of neighbors, which is decided as an optimum to be a $\mathrm{CH}$. An optimal node for $\mathrm{CH}$ selection is considered based on the good neighbored clusters, which means a neighbor with a one-hop distance or the two-hop distance. The SSOA technique considers the fitness functions like energy, alive nodes, distance and nodes coverage to obtain the optimal route among the source and the destination. After identifying the routing path, the data packets are transmitted through $\mathrm{CHs}$ in the network. If $\mathrm{CH}$ is nearer to sink or BS it transmits the data to BS or else, its transfer to another $\mathrm{CH}$ which is nearer or within its coverage. The consumption of energy is lowered by identifying the best shortest path among the source and destination. The proposed hierarchical protocol utilizes the threshold energy value that prevents leader creation groups which ensures reliable performance in the WSNs.

\section{Preliminaries}

This section provides the activities that are involved in the discussions that take place before starting with the preparation of the proposed model setup. The preliminaries include the network model structure, energy model structure, and the proposed model structure.

\subsection{Network Model}

The network model formulates based on the following criteria:

- The processing time and the initial energy for all the SNs have energy similar to the other node in WSNs.

- The distance among the sensors is computed using the Euclidean distance.

- In the sensing area, the nodes are deployed randomly in a sensor position which is deployed constantly. 
- The SNs are deployed randomly in the sensing area and the sensor positions are determined.

- The BS will receive information by selecting the $\mathrm{CHs}$ based on the SNs using a selection algorithm for $\mathrm{CH}$ that transmits energy among the $\mathrm{CHs}$ and BS.

\subsection{Energy Model}

To calculate the energy utilized among the transmitter and the receiver nodes is concluded based upon the first-order radio model. The energy consumed for transmitting and collecting the bit packets with respect to distance is calculated by using Eq. (1) and (2).

From the Equation mentioned above, $E_{\text {elec }}$ is known as the energy dissipated among the receiver-transmitter and receiver and $d_{0}$ is known as threshold distance. The threshold distance is used in equation (3) for calculation.

$$
\begin{gathered}
E_{t r}(l, d)=\left\{\begin{array}{cc}
l \times E_{\text {elec }}+l \times \varepsilon_{m p} \times d^{4} & \text { if } d>d_{0} \\
l \times E_{\text {elec }}+l \times \varepsilon_{f s} \times d^{2} & \text { if } d \leq d_{0}
\end{array}\right\} \\
E_{r c}(l, d)=l \times E_{\text {elec }} \\
d_{0}=\sqrt{\frac{\varepsilon_{f s}}{\varepsilon_{m p}}}
\end{gathered}
$$

From the Eq. (3) $\varepsilon_{f s}$ is energy amplification at the free space and $\varepsilon_{m p}$ is the amplification energy for a multi path model. These $\varepsilon_{f s}$ and $\varepsilon_{f s}$ are dependent on the amplifier model for transmission.

\section{Proposed Methodology}

The flow diagram of the proposed SSOA is shown in Fig. 1. The steps involved in the proposed method choose the $\mathrm{CHs}$ using SSOA aggregated to transmit from various member nodes directly towards the Base Station or through the CHs sequences. The selection of an optimal sensor as $\mathrm{CH}$ delivers the packets of data in an organized way offers a steady and reliable connection among the network hosts (James and Li, 2015). 


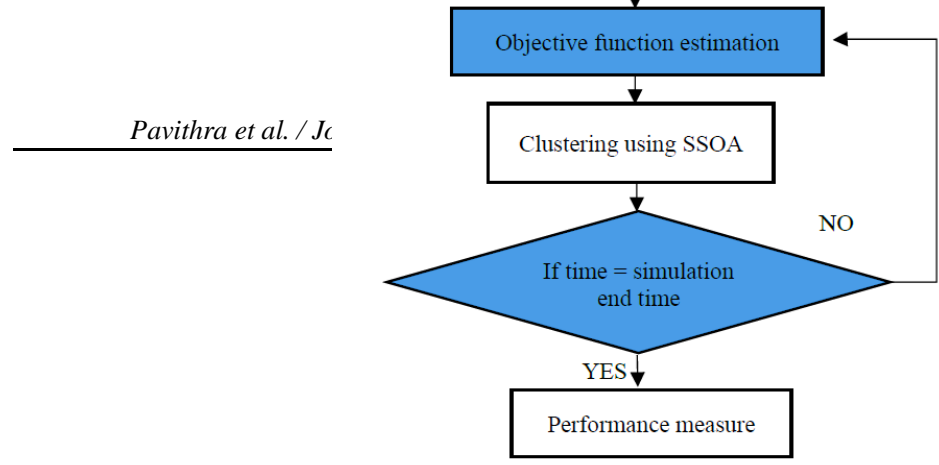

Fig. 1: The block diagram of the proposed clustering and routing spider search algorithm

\subsection{Cluster Head Selection Using SSOA}

The cluster heads are randomly selected based on one or more criteria and the selection of cluster largely affects the lifetime of WSNs. An ideal cluster head is selected based upon higher residual energy, maximization of a number of nodes, and the smallest distance from the base station. Fig. 2 shows the working of SSOA with respect to various states of spiders.

\subsubsection{Initialization of Spider Population}

Initially, the nodes are randomly deployed in the interested area. The source node and the Base Station (BS) are fixed depends on the location of the sensor nodes.

The clustering algorithm is required in WSN to divide the network into clusters where the Cluster Heads are selected from each cluster. An optimized node is selected to generate the route from $\mathrm{CH}$ to the destination (i.e., BS). If the selected node required energy to transfer the data and delivers the data from one node to another node else it selects another optimal node to transfer the information.

In SSA, the search space is formulated to overcome the problem of optimization due to the web which has hyper-dimensional space. The spider's position on the web will represent all the possible solutions for the limitation of the optimization problem. Correspondingly, the positions are updated which has feasible solutions to overcome the problem. The web present acts as a transmission media whenever the vibration is generated by spiders' movement. Each spider is present in the web which controls its position and solution is obtained based on food sources at the positions. On the web, the spiders move freely which cannot leave their positions as outside of the web and represented as the infeasible solutions for an optimization problem.

\subsubsection{Modelling of the Vibrations Through Communicable Web}

The spider moves towards the new position and the vibration generated is propagated over a web. This vibration is generated by each of the spiders hold as information whereas the other spiders present on the web will get the information based on the vibrations generated by the previous spider.

\section{Spider}

The agents are considered as spiders of SSA which performs the process of optimization. The predefined spiders are put on the web and the memory is stored by each of the $\mathrm{S}$ has the following information: 
- $\quad$ On the web, each position is represented as $\mathrm{S}$

- The information of each fitness function present in the current position is $S$

- $\quad$ From the previous iteration the target vibration from S spider

- $\quad$ The iterations occurred once in $\mathrm{S}$ changes their target vibrations

- The movement performs by $\mathrm{S}$ is based on the previous iteration

- The movement is guided by the previous iteration that employs a dimension mask.

The individual situations of the spiders $S$ describe two types of information that directs for new positions of S. Based on observation, the accurate senses of vibration are identified. The different forms of vibrations are separated and propagated on the same web which sensed the intensities respectively. The SSA generates the vibration that reaches the position and has different from the previous vibration. The vibration intensity will be correlated with the position of the fitness function. The vibration is propagated over a network that is related to the fitness of their positions. On the web, based on the vibrations the spider's senses and shares the information with other spiders is a collective social knowledge.

\section{Vibration}

Vibration is the most significant thing in the SSA that has the main character in distinguishing SSA from other metaheuristics. The SSA performs two properties that define the terms as vibration like source position, a vibration of intensity, and source intensity. The optimization problem is overcome based on the intensity of vibrations that defines the ranges from $[0,+\infty)$. The spider reaches new positions by the motions which generate the vibrations from its current position. The position of the spider is defined from time $t$ is $P_{a}(t)$ at the time argument $t$. The vibration intensity is defined as $I\left(P_{a}, P_{b}, t\right)$ that represents the position of a spider as $P_{b}$ at the time $t$ and $P_{a}$ is known as the source of the vibration is at the position. At each of the source position, a vibration intensity generated is correlated with that of the fitness positions $f\left(P_{S}\right)$ and the intensity value in Eq. (4).

$$
I\left(P_{s}, P_{s}, t\right)=\log \left(\frac{1}{f(P s)-C}+1\right)
$$

From Eq. (1) the fitness values are possible with larger fitness values is defined as $C$. The minimization problems are occurred and considered the following issues.

- The intensities obtained from the possible vibrations are positive solutions to the optimization problems.

- The positions consist of best fitness values which mean the smaller values to overcome the minimization problem shows larger intensity values compared to those of worse case of fitness values

- When a global optimum solution is obtained in terms of vibration intensity, which does not increase the vibration attenuation during the phase of malfunction.

- Over the distance, the vibration attenuates in a form of energy which is a 
physical phenomenon that accounted for the SSA designing.

\subsubsection{Search Pattern}

The SSA includes the following three phases

- Initialization phase

- Iteration phase

- Final phase

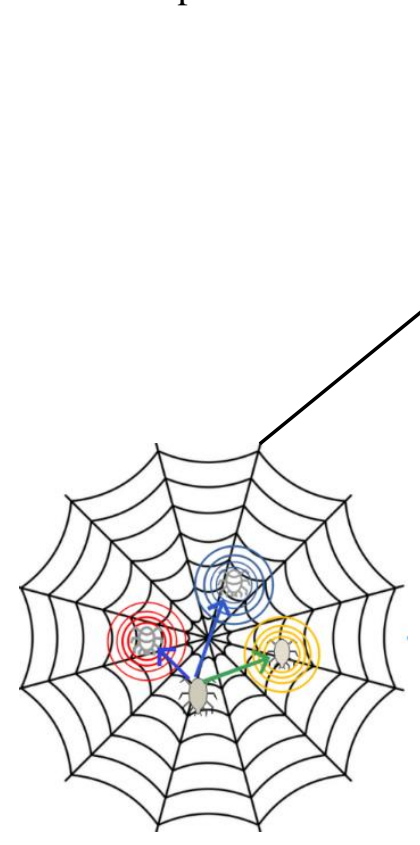

(a)

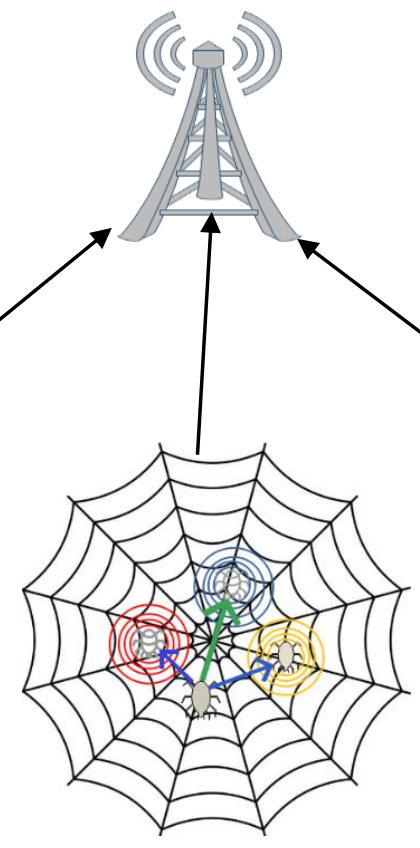

(b)

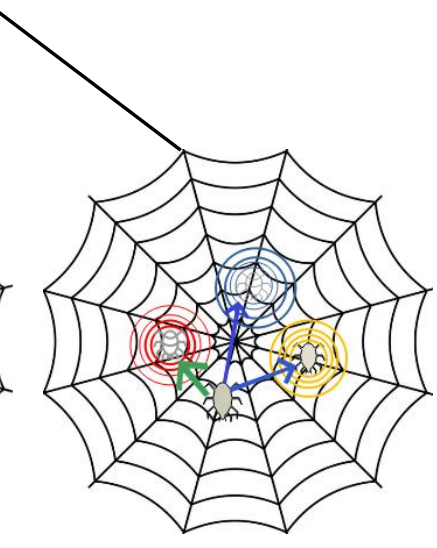

(c)

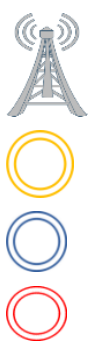

Base Station (BS)

(a) Initial position $P_{a}$ of spider $S$ and its vibration

(b) Secondary position $P_{b}$ of spider $S$ and its vibration

(c) Final Position of spider $S$ and its vibration

Fig. 2: Working of Social Spider Optimization Algorithm(a) Initial position $P_{a}$ of spider

$S$ and its vibration (b) Secondary position $P_{b}$ of spider $S$ and its vibration (c) Final Position

of spider $S$ and its vibration. 


\section{Initialization}

There are 3 phases for sequential execution and at each stage, the SSA initializes another spider iterative for obtaining solutions. At the initialization Phase, the algorithm consists of an objective function along with the solution is obtained. The SSA parameters have the values proceed further to the initial spider population for the optimization process. During the process of SSA simulations, the total number of spiders is unchanged and the fixed memory size is allocated to store the information. The spiders' positions are randomly generated in the search space to calculate the stored fitness values in the memory. Each spider in the population is set as its current positions from the initial target vibration and zero value is their vibration intensity. These attributes are initialized with zeros and stored by each spider. Thus, the initialization phases finish the process and start with another iteration phase by searching the artificial spiders' creation.

\section{Iteration phase}

The iteration phase performs and checks for a number of iterations to be executed in an algorithm and at every iteration, all spiders present on the web move for new positions for fitness values evaluation. Further, each iteration stage are divided into the sub-steps like vibration generation, evaluation of fitness functions, random walk, mask changing, and constraint handling. Initially, the fitness values present in the artificial spiders are calculated at different web positions and calculated the possible global optimum values. At each iteration, the fitness values for each spider is evaluated and generates the vibrations in their positions using the Eq. (4) and once the vibrations are generated, the simulation is propagated through the process of vibration using Euclidean Distance Eq. (5)

$$
d(p, q)=\sqrt{\sum_{i=1}^{n}\left(q_{i}-p_{i}\right)^{2}}
$$

\section{Where}

$\mathrm{p}, \mathrm{q}$ are the two points in Euclidean $\mathrm{n}$ space

$\mathrm{q}_{\mathrm{i}}, \mathrm{p}_{\mathrm{i}}$ are the Euclidean vectors starting from the origin of the space from the initial point

$\mathrm{n}$ is the $\mathrm{n}$-space

The process includes spiders $S$ that receives different vibrations from the population |pop| at different vibrations of the spiders. The information received from these vibrations constitutes from the source position and the intensity is attenuated. The vibrations from these positions generated are represented as $V$. Based on the receiver $V, S$ which selects the strongest vibration $V_{S}^{b e s t}$ from $\mathrm{V}$ that compares its intensity based on the target vibration $V^{\text {tar }}$ stored in the memory. $S$ stores $V_{S}^{\text {best }}$ as $V_{S}^{\text {tar }}$ and if in case the larger intensity of $V_{S}^{\text {best }}$ is present or if the number of iterations is larger than the target vibration, zero is reset or else the original $V_{S}^{t a r}$ is sustained by incrementing and $c_{S}$ is updated.

The source positions of $V$ and $V^{t a r}$ is represented as $P_{S}^{i}$ and $P_{S}^{t a r}$ respectively, 
and $i=\{1,2, \cdots,|p o p|\}$. The algorithm modified by $S$ to perform a random walk to move towards $V_{S}^{\text {tar }}$. A dimension mask is used to guide the movement and the spider will hold dimension mask $m$, which has the binary value from $0-1$ has a binary vector of length $\mathrm{D}$ which is known as dimension $\mathrm{D}$ that overcomes the problem of optimization. The values present in the mask are zero where the values are set initially to each of the spider constitutes of probability as $1-p_{c}^{c_{S}}$ where $p_{c} \in(0,1)$ for defining the attributes describe the mask changing probability. For each bit, the vector probability $p_{m}$ is assigned as 1 and $1-p_{m}$ should be set as zero. The $p_{m}$ the term is known as a user-controlled parameter ranging from $(0,1)$. The mask bit of each bit is independently changed and has not relation corresponding to the previous mask. If the zeros are present in all bits, one random value from the mask is changed as one. Likewise, the zero value is replaced in random bit assigned the values are zero. New following positions are generated which has mass function is now defined as $P_{S}^{f 0}$ based on $S$ mask. The value for $i^{\text {th }}$ dimension at the position $P_{\varsigma}^{f 0}$ is calculated by using Equation (6).

$$
P_{S, i}^{f 0}=\left\{\begin{array}{cc}
P_{S, i}^{t a r} & m_{s, i}=0 \\
P_{S, i}^{r} & m_{s, i}=1
\end{array}\right\}
$$

$r$ is known as the random integer value generated in the range of $[1,|p o p|], m_{s, i}$ stands with the $i^{t h}$ dimension having the dimension mask as $m$ for each of the $S$. Here $r$ is used for two different dimensions that are having $m_{s, i}=1$ independently utilized and generated. The generated $P_{S}^{f 0}$ includes $S$ random walk at each position to performs using the following Equation (7):

$$
P_{S}(t+1)=P_{S}+\left(P_{S}-P_{S}(t-1)\right) \times r+\left(P_{S}^{f 0}-P_{S}\right) \odot R
$$

The aforementioned equation (4) represents the element-wise multiplication, $R$ is random float-point vector numbers that are generated ranging from 0 to 1 uniformly. In $P_{S}^{f 0}, S$ will move initially along with the previous direction and the direction is based on the movement of the previous iteration. Once after the random walk, the spider stores their movement in the current iteration so that would be helpful for the next iteration. Finally, it ends with the sub-step generated from the random walk.

\section{Final Sub-Step}

The final sub-step is a constraint that is generated to handle at each iteration phase. The spiders move from the web at the time of the random walk process caused optimization problems and violated the constraints. The boundary constraints are handled by other literature which uses a random approach to reflect and absorbs the approaches widely by adopting the methods. The present research adopts to reflect the approach to boundary constraining which produces the boundary constraint at free position is performed by using the Equation (8).

$$
P_{S}(t+1) b y
$$




$$
P_{s, i}(t+1)=\left\{\begin{array}{l}
\left(\overline{x_{l}}-P_{s, i}\right) \times r \text { if } P_{s, i}(t+1)>\overline{x_{l}} \\
\left(P_{s, i}-\underline{x_{i}}\right) \times r \text { if } P_{s, i}(t+1)<\underline{x_{i}}
\end{array}\right\}
$$

From the equation $\overline{x_{l}}$ is known as the upper bound present in a search space for the $i^{\text {th }}$ dimension, the lower bound is represented as $x_{i}$ for the corresponding dimension. Random floating point $r$ is ranging from $(0,1)$.

The stopping criteria are required to be matched until the iteration phase loops are met. The criteria for stopping the iteration are concluded once the maximum number of iterations is reached. Once CPU time is utilized up to the maximum level, then the error rate generated reaches a maximum number of iteration. The iteration phase generates the best solution for the best fitness function and these three phases complete the SSA algorithm with the following pseudo-code is shown in algorithm 1 .

The fitness function obtains the best solution based on the objective function which is particularly obtained to summarizes the best solution that gives a closer solution for the constructed design. The fitness function obtained from the SSA algorithm to the sensor networks for the selection of $\mathrm{CH}$. The dead nodes generate residual energy generated to avoid the fitness function during the process of clustering. The distance among the nodes starts from $\mathrm{CH}$ to $\mathrm{BS}$ which is used for the selection of $\mathrm{CH}$ optimally minimizes consumption of energy from the nodes. The distance generated among the node degree for the $\mathrm{CH}$ selection has normal nodes preserved a higher number of nodes. The cluster members have a higher centrality that leads to the distance transmissions among the $\mathrm{CH}$ members to the $\mathrm{CH}$.

\section{Pseudocode for SSOA}

Appoint qualities to the parameters of SSOA.

Make the population of spiders pop and allot memory for them.

$V_{S}^{\text {tar }}$ for every bug is initialized

while stopping rules not met do

for every spider $S$ in $p o p$ do

Assess the fitness worth of $S$

Produce a vibration at the situation of $S$

\section{end for}

for every spider $S$ in $p o p$ do

Figure the intensity of the vibrations $V$ created by all spiders.

Select the most grounded vibration $V_{S}^{b e s t}$ from .

If $V_{S}^{\text {best }}$ is larger than $V_{S}^{\text {tar }}$

\section{end if}

$$
\text { Store } V_{S}^{\text {best }} \text { as } V_{S}^{\text {tar }} \text {. }
$$

Update cs.

Produce an arbitrary number $r$ from $[0,1)$.

$$
\text { if } r>p_{c}^{c_{S}} \text { then }
$$


end if

Update the measurement veil

\author{
Generate $P_{S, i}^{f 0}$ \\ Perform a random walk. \\ Address any violated constraints.
}

\title{
end for \\ end while
}

Output the best solution found.

The fitness function considered for SSA is described as shown below :

\section{a. Residual energy}

The energy generated from the $\mathrm{CH}$ performed tasks for collecting the data from the normal sensor nodes to the BS during the data transmission. High energy is required by $\mathrm{CH}$ for task accomplishment and aforementioned tasks for higher energy are accomplished to perform the tasks. Therefore, the node which has high energy accomplishes the tasks and the node which has higher residual energy is referred to as $\mathrm{CH}\left(f_{1}\right)$. The residual energy is represented using the below Eq. (9)

$$
f_{1}=\sum_{i=1}^{m} \frac{1}{E_{C H_{i}}}
$$

From Eq. (9) the residual energy is represented as $E_{C H}$ obtained from the cluster head $i$ which is represented as $E_{C H_{i}}$.

\section{b. Distance between the sensor nodes}

The distance between the normal and SNs from their $\mathrm{CH}$ mainly depends on the distance transmission path generated from the node having a lesser transmission distance from the BD. The distance between the normal sensor to that of the $\mathrm{CH}$ $\left(f_{2}\right)$ is expressed in equation (10).

$$
f_{2}=\sum_{j=1}^{m} \frac{\sum_{i=1}^{I_{j}} \operatorname{dis}\left(s_{i}, C H_{j}\right)}{I_{i}}
$$

From the Eq. (7), the distance obtained between the sensor $i$ and $C H_{i}$ is represented as $d\left(s_{i}, \mathrm{CH}_{i}\right)$ where the sensor nodes belong to $\mathrm{CH}$ is represented as $I_{i}$

\section{c. Distance from the $\mathrm{CH}$ to the $\mathrm{BS}$}

The distance between the $\mathrm{BS}$ and $\mathrm{CH}$ depends upon the node energy consumption amount used through the transmission path. The BS is placed far away from the $\mathrm{CH}$ which instantly requires more energy to process the data transmission. Thus, the lesser distance obtained from the BS is utilized to transmit the data. Eq. (11) calculates the objective function which means it calculates the distance among the $\mathrm{BS}\left(f_{3}\right)$ and $\mathrm{CH}$.

$$
f_{3}=\sum_{i=1}^{m} \operatorname{dis}\left(\mathrm{CH}_{j}, \mathrm{BS}\right)
$$

\section{d. Node degree}

The number of sensor nodes belongs to their respective $\mathrm{CH}$ that defines the node 
degree. If the number of sensors present is lesser than that of selected ones, then $\mathrm{CHs}$ having higher cluster member which lowers the energy consumption within less duration of time. In the Eq. $(12),\left(f_{4}\right)$ is expressed as a node degree as shown below :

$$
f_{4}=\sum_{i=1}^{m} I_{i}
$$

where $I_{i}$ are a number of sensor nodes belonging to.

\section{e. Node centrality}

The node centrality is defined as the distance of a node that is centrally stayed from that of the neighboring nodes is expressed using the Eq. (13)

$$
f_{5}=\sum_{i=1}^{m} \frac{\sqrt{\left(\sum_{j \in n} \operatorname{dist}^{2}(i, j)\right) / n(i)}}{\text { Network dimension }}
$$

From the Eq. (10), $n(i)$ is known as the neighboring node from the $C H_{i}$.

For each of the objective value, the weight functions obtained are aggregated and the Eq. (14) is expressed as shown as follows:

$$
\text { fitness }=f_{1}+f_{2}+f_{3}+f_{4}+f_{5}
$$

Once the path is created from the source to the destination, the data is transmitted from the source node to the destination node. An optimal path is developed among the objective functions that consider the parameters like residual energy between the nodes, the distance among the nodes, and the objective functions. This manages the residual energy present in the nodes that avoid in a network. The proposed method performances are analyses in terms of Percentage of nodes alive, Percentage of nodes dead, Energy consumption and Total packet sent.

\section{Performance Evaluation}

\section{Alive Nodes:}

The Alive nodes are the total number of nodes alive in the network

\section{Average Energy Consumption:}

The energy consumed by each node during the iterations is known as Average Energy Consumption.

\section{Dead Nodes:}

The two sensors node is communicated directly within one communication that hops one after the other. Some of the regions present in the network have dead nodes that are a part of network coverage which refers that the data are unavailable. The protocol behavior is observed which consumes the energy during the data transmission and the nodes deplete the energies until they are dead completely.

\subsection{Simulation Setup}

The comparative and simulation analysis is performed to assume the total number 
of nodes of 2000. The nodes present are homogeneous that is selected randomly. The BS is fixed at the center from the sensing area of about $500 \mathrm{~m}^{2}$. The signal collision and interference are taken into consideration with the initial energy of the node is $0.5 \mathrm{~J}$. The clusters are arranged in a network hierarchic where the $\mathrm{CH}$ will fuse data reduces the correlation and redundancy to be considered as a static node. The performance and experimental setup of the proposed SSOA method are discussed in table 1 .

Table 1: Parameters for the process of simulation

\begin{tabular}{|c|c|}
\hline Parameters & Value \\
\hline Area & $500 \mathrm{~m}^{2}$ \\
\hline The energy of the node & $0.5 \mathrm{~J}$ \\
\hline Number of Rounds & 2000 \\
\hline Cluster percentage & 0.3 \\
\hline
\end{tabular}

The cluster based on optimization process which analyses the various metrics like dead nodes, alive nodes, the average consumption of energy. The performance measures are explained as follows:

\subsection{Performance Evaluation in Terms of Average Energy and Total Energy}

The SSA protocol reduces the energy in a network as per the data transmission process and is quite important for observing the network's behavior. When the number of rounds is increased the remained energy is also better. The number of rounds is covered with the process of data was remained with the energy consumption showed that the proposed protocol is better when compared to other protocols for the expanded network area. The energy node at each round preserves the minimum consumption of energy resulted from the dual-hop communication. Table 2 shows the results obtained for the proposed SSOA in terms of Average and total Energy (J). Fig. 3 shows the graphical representation for the proposed SSOA in terms of total Energy and Fig. 4 shows the graphical representation for the proposed SSOA in terms of Average Energy.

Table 2: Results evaluation for the proposed SSOA in terms of Average and total

Energy $(\mathrm{J})$

\begin{tabular}{|c|c|c|}
\hline Rounds & Average Energy (J) & Total Energy(J) \\
\hline 0 & 0.99999 & 9.99993 \\
\hline 10 & 0.99991 & 9.99914 \\
\hline 20 & 0.99984 & 9.99838 \\
\hline 30 & 0.99976 & 9.99763 \\
\hline 40 & 0.99969 & 9.99687 \\
\hline 50 & 0.99961 & 9.99611 \\
\hline 60 & 0.99954 & 9.99535 \\
\hline 70 & 0.99946 & 9.99459 \\
\hline
\end{tabular}




\begin{tabular}{|c|c|c|}
\hline 80 & 0.99938 & 9.99383 \\
\hline 90 & 0.99931 & 9.99308 \\
\hline 100 & 0.99923 & 9.99232 \\
\hline 110 & 0.99916 & 9.99156 \\
\hline 120 & 0.99908 & 9.9908 \\
\hline 130 & 0.999 & 9.99004 \\
\hline 140 & 0.99893 & 9.98928 \\
\hline 150 & 0.99885 & 9.98853 \\
\hline 160 & 0.99878 & 9.98777 \\
\hline 170 & 0.9987 & 9.98701 \\
\hline 180 & 0.99863 & 9.98625 \\
\hline 190 & 0.99855 & 9.98549 \\
\hline 200 & 0.99847 & 9.98473 \\
\hline
\end{tabular}

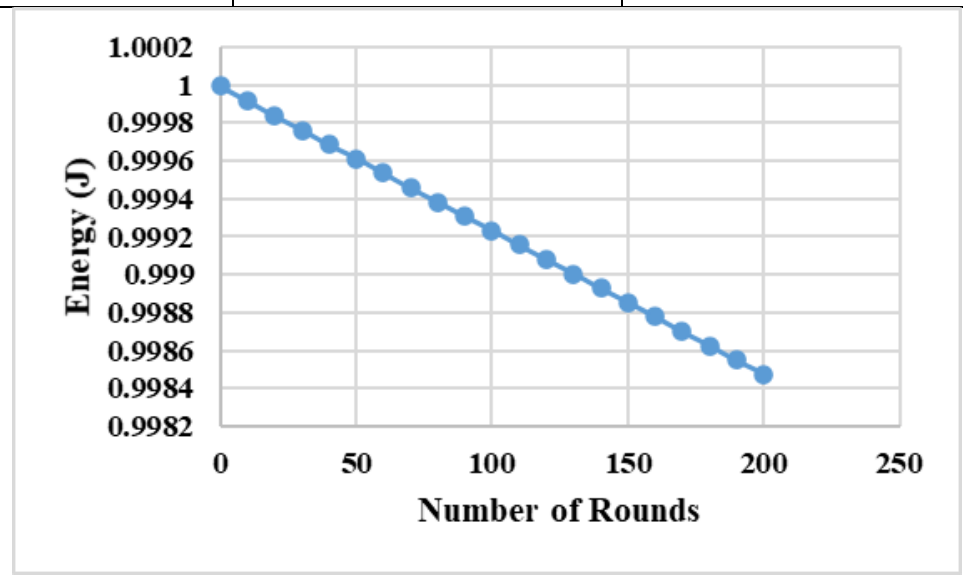

Fig. 3: The graphical representation for the proposed SSOA in terms of total Energy

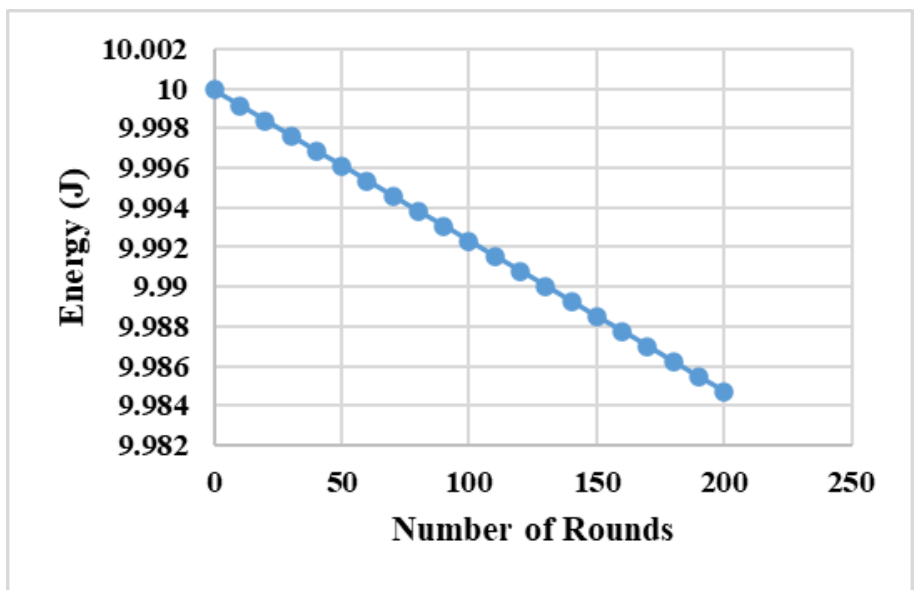

Fig. 4: The graphical representation for the proposed SSOA in terms of Average Energy 


\subsection{Performance Evaluation of Number of Dead Nodes at Each Iteration and the Cluster (\%)}

The proposed SSOA evaluates the number of nodes for all smaller rounds to the total number of the present dead node. The last node present in a network defines the network lifetime improvement is as shown in table 3. Fig. 5 shows the graphical representation for the proposed SSOA in terms of the Number of Dead Nodes.

Table 3: Results evaluation for the proposed SSOA in terms of Number of dead nodes and clustering percentage

\begin{tabular}{|c|c|c|}
\hline No. of Rounds & No. of dead nodes & Clustering (\%) \\
\hline 0 & 7 & 35 \\
\hline 10 & 3 & 15 \\
\hline 20 & 3 & 15 \\
\hline 30 & 3 & 15 \\
\hline 40 & 3 & 15 \\
\hline 50 & 3 & 15 \\
\hline 60 & 3 & 15 \\
\hline 70 & 3 & 15 \\
\hline 80 & 3 & 15 \\
\hline 90 & 3 & 15 \\
\hline 100 & 3 & 15 \\
\hline 110 & 3 & 15 \\
\hline 120 & 3 & 15 \\
\hline 130 & 3 & 15 \\
\hline 140 & 3 & 15 \\
\hline 150 & 3 & 15 \\
\hline 160 & 3 & 15 \\
\hline 170 & 3 & 15 \\
\hline 180 & 3 & 15 \\
\hline 190 & 3 & 15 \\
\hline 200 & 3 & 15 \\
\hline & & \\
\hline & & \\
\hline
\end{tabular}

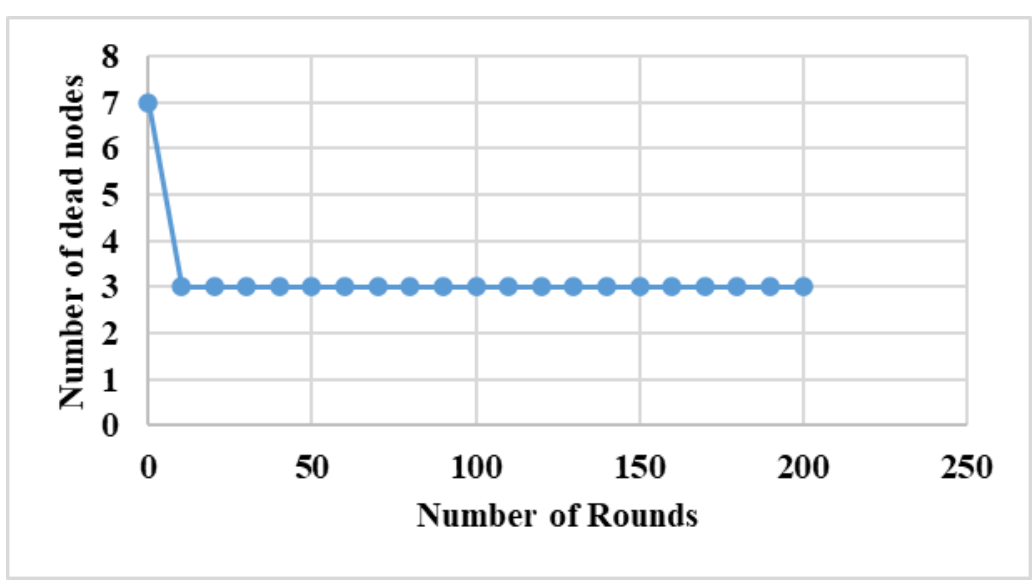

Fig. 5: The graphical representation for the proposed SSOA in terms of the Number of Dead 
Nodes

\subsection{Performance Evaluation of Clustering Percentage (\%)}

Fig. 6 shows the $\mathrm{CH}$ selection process during the process of experimentation is carried out when the count of nodes reaches 190 . The node variations which range from 100 to 200 have the node distribution. The $\mathrm{CH}$ selection is completed to the process of the proposed network is shown in Fig. 6.

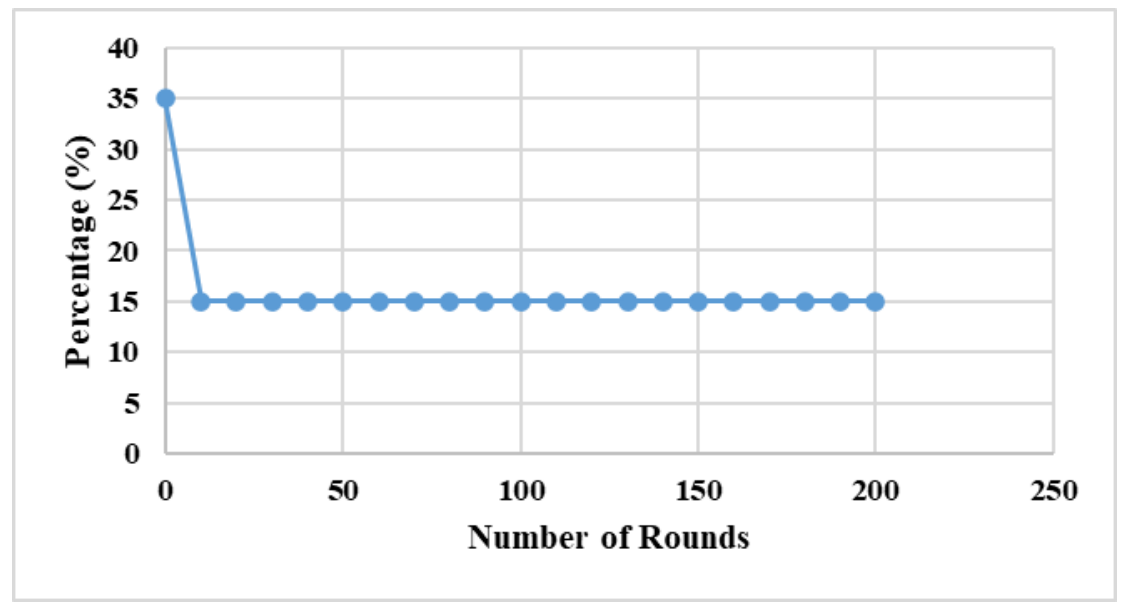

Fig. 6: The graphical representation for the proposed SSOA in terms of Clustering Percentage (\%)

\subsection{Comparative Analysis}

The developed GAPSO-H showed average energy consumption as $16 \mathrm{~J}$ for a significant level. Similar performances were achieved for the remaining algorithms that are resulted and showed the worst performance. Fig. 6 shows that the plot for the remained energy with respect to each of the algorithms. The dead nodes present is shown in the $\mathrm{x}$-axis where the number of rounds present is shown in the $\mathrm{y}$-axis. When the data label was added for each of the algorithms, the GAPSO-H showed better performances compared to other optimization algorithms. Similarly, the hybrid PSO-GWO became Nil when the developed approach reaches 500 rounds with respect to the alive sensor nodes and the number of rounds reached up to 1000 th to 1500 th rounds. The improvement is seen as it chooses cluster head to reduce more energy consumption instead of wasting the possibility of losing energy is improved. The developed SSOA provided better conservation of energy for less number of rounds because the number of rounds increased with respect to packet delivery ratio. The residual energy and other parameters increase the network lifetime of the overall performance is increased. As the multiple parameters for the $\mathrm{CH}$ selection are done when the conservation of energy in the network is better when compared to the existing optimization approaches. The proposed SSOA 
achieved better performances for energy consumption with respect to the dead nodes that utilized less energy when compared to the existing models. Table 4 shows the Comparison of the proposed SSOA with respect to the existing GAPSO Hybrid PSO-GWO, and MW-LEACH algorithms. Fig. 7 shows the Comparative analysis for the proposed SSOA and the existing researches.

Table 4: Comparison of proposed SSOA with respect to the existing GAPSO Hybrid PSO-GWO, and MW-LEACH algorithms

\begin{tabular}{|c|c|c|c|}
\hline Methodologies & Number of rounds & Dead nodes & Energy Consumption (J) \\
\hline $\begin{array}{c}\text { Sahoo et al. } \\
(2021)\end{array}$ & 500 & 2 & 20 \\
\cline { 2 - 4 } & 1000 & 10 & 16 \\
\cline { 2 - 4 } & 1500 & 70 & 14 \\
\hline \multirow{2}{*}{$\begin{array}{c}\text { El Khediri et } \\
\text { al. (2020) }\end{array}$} & 500 & 200 & - \\
\cline { 2 - 4 } & 1000 & 201 & - \\
\hline \multirow{2}{*}{$\begin{array}{c}\text { Prithi et al. } \\
(2021)\end{array}$} & 1500 & 202 & - \\
\cline { 2 - 4 } & 500 & 600 & 610 \\
\hline \multirow{2}{*}{\begin{tabular}{c} 
Proposed \\
\cline { 2 - 4 }
\end{tabular}} & 1000 & 620 & 600 \\
\cline { 2 - 4 } & 1500 & 640 & 0.996199 \\
\cline { 2 - 4 } & 500 & 3 & 0.992406 \\
\hline
\end{tabular}

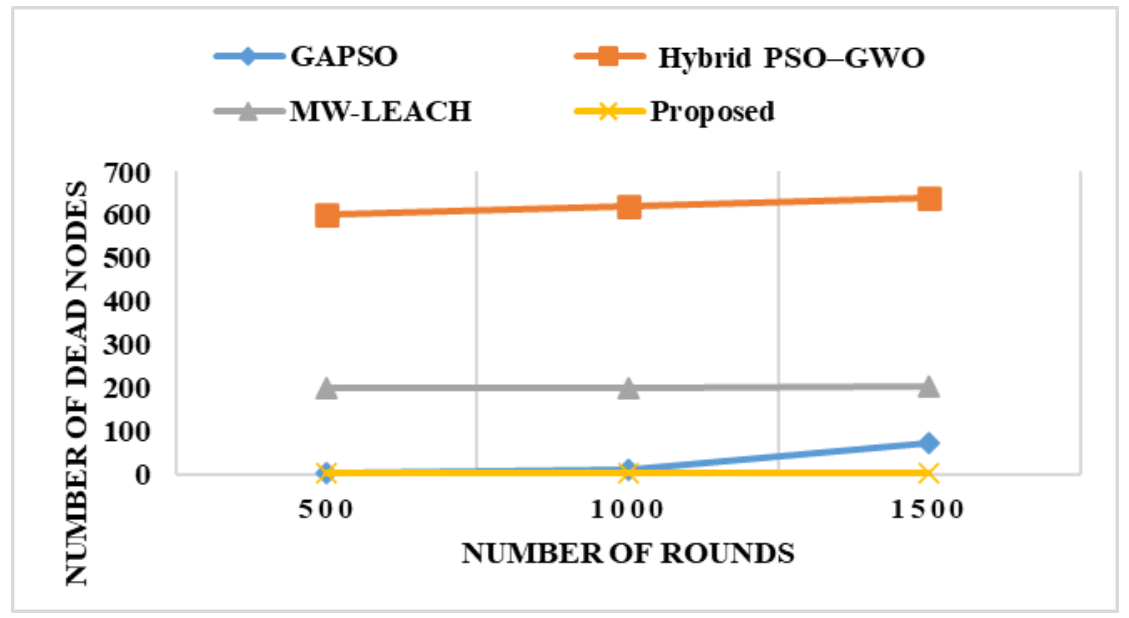

Fig. 7: Comparative analysis for the proposed SSOA and the existing researches

The results showed that the proposed SSOA has shown improvement in the performance when compared with the existing GAPSO-H, MW-LEACH state-ofthe-arts algorithms. 


\section{Conclusion}

In the present research work, the proposed SSOA performs the $\mathrm{CH}$ selection based on the residual energy present among the other CHs. The selection of cluster points is done based on the initial points that were closer to that of the $\mathrm{CH}$ having the density function. The experimental evaluation of the proposed SSOA approach is outperformed when compared to the Hybrid PSO-GWO, GA-PSO, and MWLEACH that evaluated the results Dead nodes, energy consumption, average energy consumption, and Cluster percentage. The proposed SSOA showed improvement in energy consumption of $20 \%$ when compared to the existing GAPSO-H, MWLEACH. However, the present research work should build on a real testbed of sensors for specific domain applications. In the future, the multiple sink mobility scenarios using the optimization approaches can be examined for better results.

\section{References}

Boveiri, H.R. (2020). An enhanced cuckoo optimization algorithm for task graph scheduling in cluster-computing systems. Soft Computing, 24(13), 10075-10093.

El Khediri, S., Khan, R.U., Nasri, N. and Kachouri, A. (2020). Energy efficient adaptive clustering hierarchy approach for wireless sensor networks. International Journal of Electronics, 1-20.

Ezhilarasi, M. and Krishnaveni, V. (2019). An evolutionary multipath energyefficient routing protocol (EMEER) for network lifetime enhancement in wireless sensor networks. Soft Computing, 23(18), 8367-8377.

Han, Y., Byun, H. and Zhang, L. (2020). Energy-Balanced Cluster-Routing Protocol Based on Particle Swarm Optimization with Five Mutation Operators for Wireless Sensor Networks. Sensors, 20(24), 7217.

Han, Y., Zhang, K., Li, H., Coelho, E.A.A. and Guerrero, J.M. (2017). MAS-based distributed coordinated control and optimization in microgrid and microgrid clusters: A comprehensive overview. IEEE Transactions on Power Electronics, 33(8), 64886508 .

Haseeb, K., Bakar, K.A., Abdullah, A.H. and Darwish, T. (2017). Adaptive energy aware cluster-based routing protocol for wireless sensor networks. Wireless Networks, 23(6), 1953-1966.

James, J.Q. and Li, V.O., 2015. A social spider algorithm for global optimization. Applied Soft Computing, 30, pp.614-627. 
Jemal, A.F., Hussen, R.H., Kim, D.Y., Li, Z., Pei, T. and Choi, Y.J. (2018). Energyefficient selection of cluster headers in wireless sensor networks. International Journal of Distributed Sensor Networks, 14(3), 1550147718764642.

Jha, S.K. and Eyong, E.M. (2018). An energy optimization in wireless sensor networks by using genetic algorithm. Telecommunication Systems, 67(1), 113-121.

Maheshwari, P., Sharma, A.K. and Verma, K. (2021). Energy efficient cluster based routing protocol for WSN using butterfly optimization algorithm and ant colony optimization. Ad Hoc Networks, 110, p.102317.

Mehta, D. and Saxena, S. (2020). MCH-EOR: Multi-objective cluster head based energy-aware optimized routing algorithm in wireless sensor networks. Sustainable Computing: Informatics and Systems, 28, 100406.

Pitchaimanickam, B. and Murugaboopathi, G. (2020). A hybrid firefly algorithm with particle swarm optimization for energy efficient optimal cluster head selection in wireless sensor networks. Neural Computing and Applications, 32(12), 77097723 .

Preethiya, T., Muthukumar, A. and Durairaj, S., 2020. Double cluster head heterogeneous clustering for optimization in hybrid wireless sensor network. Wireless Personal Communications, 110(4), 1751-1768.

Prithi, S. and Sumathi, S. (2021). Automata Based Hybrid PSO-GWO Algorithm for Secured Energy Efficient Optimal Routing in Wireless Sensor Network. Wireless Personal Communications, 117(2), 545-559.

Rao, P.S., Jana, P.K. and Banka, H. (2017). A particle swarm optimization based energy efficient cluster head selection algorithm for wireless sensor networks. Wireless networks, 23(7), 2005-2020.

Sahoo, B.M., Pandey, H.M. and Amgoth, T. (2021). GAPSO-H: A hybrid approach towards optimizing the cluster based routing in wireless sensor network. Swarm and Evolutionary Computation, 60, 100772.

Singh, S., 2020. An energy aware clustering and data gathering technique based on nature inspired optimization in WSNs. Peer-to-Peer Networking and Applications, 13(5), 1357-1374.

Wang, Y. and Wang, Z., 2019. Routing algorithm of energy efficient wireless sensor network based on partial energy level. Cluster computing, 22(4), 8629-8638. 
Ya-qiong, Z. and Yun-rui, L., 2016. A routing protocol for wireless sensor networks using K-means and Dijkstra algorithm. International Journal of Advanced Media and Communication, 6(2-4), 109-121.

Zhang, J., Glezakou, V.A., Rousseau, R. and Nguyen, M.T., 2020. NWPEsSe: an adaptive-learning global optimization algorithm for nanosized cluster systems. Journal of chemical theory and computation, 16(6), 3947-3958. 